\title{
A New Robust Output Feedback Variable Structure Controller for Uncertain More Affine Nonlinear Systems with Mismatched Uncertainties and Matched Disturbance
}

\author{
Jung-Hoon Lee ${ }^{* \star}$
}

\begin{abstract}
In this note, a new robust nonlinear output feedback variable structure controller is first systematically and generally designed for the output control of more affine uncertain nonlinear systems with mismatched uncertainties and matched disturbance. A transformed integral output feedback sliding surface with a most simple form is applied in order to remove the reaching phase problems. The closed loop exponential stability and the existence condition of the sliding mode on the integral output feedback sliding surface is investigated with a corresponding output feedback control input in Theorem 1. For practical application the continuous implementation of the control input is made by the modified saturation function. The effectiveness of the proposed controller is verified through a design example and simulation study.
\end{abstract}

Key words: output feedback, variable structure system, sliding mode control, mismatched uncertainties, nonlinear system

\section{Introduction}

Stability analysis and controller design for uncertain nonlinear systems is open problem now[1]. So far numerous design methodologies exist for the controller design of nonlinear systems[2]. Among them the sliding mode control(SMC) can provide the effective means to the problem of controlling uncertain nonlinear systems[15][16][17]. Toledo and Linares applied the sliding mode approach to the nonlinear output regulator scheme[19]. The key concept is designing sliding submanifold with the zero tracking error submanifold. Bartolini and Ferrara proposed a simplex of control vectors for

*ERI, Dept. of Control \& Instrum. Eng., Gyeongsang National University,

$\star$ Corresponding author, email:jhleew@gnu.ac.kr, phone:+82-55-772-1742

Manuscript received Feb. 23 2014; reviced:June 02 2014; accepted June 032014 multi input uncertain nonlinear systems[20]. Lu and Spurgeon considered the robustness of dynamic sliding mode control of nonlinear system which are in differential input-out form with additive uncertainties in the model[21]. Bartolini, Pisano, and Usai analyzed the digital implementation of a second-order sliding mode control scheme for uncertain nonlinear system[22]. Flemming surveyed so called soft variable structure controls, compared them to other[23], Wang, Jiang, Zhou, and Gao proposed a switching control law between a first order sliding mode control and a second order sliding mode control[24]. Tang, Dong, Gao suggested the optimal SMC for nonlinear system with time-delay[25]. Pan, Kumar, Liu, and Furuta designed the nonlinear time varying sliding sector for a single input nonlinear time varying input affine system in the form of state dependent linear time variant system with matched uncertainties[26]. Lee reported the systematic design of the SMC for 
uncertain affine nonlinear system with mismatched uncertainties and matched disturbance[30]. Lee introduced the integral action to the nonlinear VSS with mismatched uncertainties and matched disturbance to improve the performance[31][32].

Until now, a nonlinear output feedback controller design for uncertain nonlinear systems with mismatched uncertainties and matched disturbance is rarely presented.

In this technical note, a first systematic general design of a new nonlinear output feedback variable structure controller is presented for the output control of uncertain affine nonlinear systems with mismatched uncertainties and matched disturbances. An transformed output feedback integral sliding surface is applied in order to remove the reaching phase. The closed loop exponential stability and the existence condition of the sliding mode on the output feedback integral sliding surface is investigated with a corresponding control input in Theorem 1. For practical application the continuous implementation of the control input is made by the modified saturation function The effectiveness of the proposed nonlinear output feedback VSS controller is verified through a design example and simulation study.

\section{A Nonlinear Output feedback Variable Structure Systems}

\section{Description of plants}

Consider an affine uncertain nonlinear system

$$
\begin{aligned}
& \dot{X}=f^{\prime}(X, t)+g(X, t) u+\bar{d}(X, t), \quad X(0) \\
& Y=C \cdot X(t), \quad Y(0)=C \cdot X(0)
\end{aligned}
$$

where $X \in R^{n}$ is the state, $X(0)$ is its initial state, $Y \in R^{q}, q \leq n$ is the output, $Y(0)$ is an initial condition of the output $u \in R^{1}$ is the control to be determined, mismatched uncertainty $f^{\prime}(X, t) \in C^{k}$ and $\quad g(X, t) \in C^{k}, k \geq 1, \quad g(X, t) \neq 0$, for all $X \in R^{n}$ and for allt $\geq 0$ are of suitable dimensions, and $\bar{d}(x, t)$ implies bounded matched external disturbances.

Assumption[26]

$\mathrm{A} 1: f^{\prime}(X, t)$ is continuously differentiable and $f^{\prime}(0, t)=0$ for all $t \geq 0$.

Then, uncertain nonlinear system (1) can be represented in more affine nonlinear system of state dependent coefficient form[26]-[28]

$$
\begin{aligned}
& \dot{X}= f(X, t) X+g(X, t) u+\bar{d}(X, t), \quad X(0) \\
&= {\left[f_{0}(X, t)+\Delta f(X, t)\right] X } \\
&+\left[g_{0}(X, t)-\Delta g(X, t)\right] u+\bar{d}(X, t) \\
&= f_{0}(X, t) X+g_{0}(X, t) u+d(X, t) \\
& Y=C \cdot X(t) \\
& d(X, t)=\Delta f(X, t) X-\Delta g(X, t) u+d^{\prime}(X, t)
\end{aligned}
$$

where $f_{0}(X, t)$ and $g_{0}(X, t)$ is each nominal value such that $f^{\prime}(X, t)=\left[f_{0}(X, t)+\triangle f(X, t)\right] X \quad$ and $g(X, t)=\left[g_{0}(X, t)-\triangle g(X, t)\right]$, respectively, $\triangle f(X, t)$ and $\triangle g(X, t)$ are matched or mismatched uncertainties, and $d(X, t)$ is the mismatched lumped uncertainties.

Assumption:

A2: The nominal input matrix $g_{0}(X, t)$ is constant, i.e, $g_{0}(X, t)=B$

A3: The pair $\left(f_{0}(X, t), B\right)$ is controllable and $\left(f_{0}(X, t), C\right)$ is observable for all $X \in R^{n}$ and for all $t \geq 0$

A4:unmatched $\triangle f(X, t)$, matched $\Delta g(X, t)$, and matched $d^{\prime}(X, t)$ are unknown and bounded and are satisfied by the following conditions

$$
\begin{gathered}
\triangle f(X, t)=\Delta f^{\prime}(X, t) C^{T} C=\Delta f^{\prime \prime}(X, t) C \\
\triangle g(X, t)=B B^{T} \triangle g^{\prime}(X, t)=B \Delta I, \\
\triangle I \leq p<1, \quad 1>p>0 \\
d(X, t)=B B^{T}(X, t) d^{\prime}(X, t)=B d^{\prime \prime}(X, t)
\end{gathered}
$$

A5: The lumped uncertainties $d(x, t)$ is bounded A6: $\ddot{X}$ is bounded if $\dot{u}$ and $\dot{d}^{\prime}(x, t)$ is bounded.

Now, the integral of the output is augmented as follows:

$$
\begin{aligned}
& \dot{Y}_{0}=A_{0} Y(X(t)), \quad Y_{0}(0) \\
& Y_{0}(t)=A_{0} \int_{0}^{t} Y(X(\tau)) d \tau+Y_{0}(0)
\end{aligned}
$$

where $Y_{0} \in R^{r}, \quad r \leq q$ is the integral of the output and $Y_{0}(0)$ is the initial condition of the integral state determined later, and $A_{0}$ is appropriately dimensioned without loss of generality, $A_{0}=I$.

2. Transformed output feedback integral sliding surface

\section{Assumption}

A7: $\left(H_{1} C B\right)$ has the inverse for some non zero row vector $H_{1}$.

Now, a transformed output feedback integral sliding surface is suggested be [33]

$$
S=\left(H_{1} C B\right)^{-1}\left(H_{1} \cdot Y+H_{0} \cdot Y_{0}\right)(=0)
$$




$$
Y_{0}(0)=-H_{0} H_{1} \cdot Y(0)
$$

where $H_{0}^{-}=\left(H_{0}^{T} W H_{0}\right)^{-1} H_{0}^{T} W$. In (8), non zero row vector $H_{1}$ and $H_{0}$ are the design parameters satisfying the following relationship

$$
\begin{aligned}
& H_{1} C\left[f_{0}(X, t)-B K(Y) C\right]+H_{0} C \\
& =H_{1} C A_{C}+H_{0} C=0
\end{aligned}
$$

where closed loop system matrix $A_{C}=f_{0}(X, t)-B K(Y) C$ and $K(Y)$ is an output feedback gain. At $t=0$, this output feedback integral sliding surface is zero so that there is no reaching phase[32]. In (8), $H_{1}$ and $H_{0}$ are non zero row vectors as the design parameter such that the following assumption is satisfied.

Assumption

A8: $H_{1} g(X, t)$ and $H_{1} B$ have the full rank and are invertible

The equivalent control input is obtained using $\dot{s}=0$ [15] as

$$
\begin{aligned}
u_{e q}= & -\left[H_{1} C g(X, t)\right]^{-1} H_{1} f(X, t) X \\
& -\left[H_{1} C g(X, t)\right]^{-1} \bar{d}(X, t) \\
& -\left[H_{1} C g(X, t)\right]^{-1} H_{0} Y_{0}
\end{aligned}
$$

This control input can not be implemented because of the uncertainties and disturbances. The ideal sliding mode dynamics of the output feedback integral sliding surface (8) can be derived by the equivalent control approach ${ }^{[16]}$ as

$$
\begin{aligned}
\dot{X}_{s}= & {\left[f_{0}(X, t)\right.} \\
& -B\left(H_{1} C B\right)^{-1} H_{1} f_{0}(X, t) \\
& \left.-B\left(H_{1} C B\right)^{-1} H_{0} C\right] X_{s}, \\
& X_{s}(0)=X(0) \\
Y_{s}= & C \cdot X_{s}
\end{aligned}
$$

and from $\dot{s}=0$, the another ideal sliding mode dynamics is obtained as ${ }^{[30]}$

$$
\dot{Y}_{s}=-H_{1}^{-} H_{0} Y_{s}, \quad Y_{s}(0)
$$

where $H_{1}^{-}=\left(H_{1}^{T} W H_{1}\right)^{-1} H_{1}^{T} W$. The solution of (12) or (14) identically defines the output feedback integral sliding surface. Hence to design the output feedback integral sliding surface as stable, this ideal sliding dynamics (9) is designed to be stable. To choose the stable gain based on the Lyapunov stability theory, the ideal sliding dynamics (9) is represented by the nominal plant of (2) as

$$
\begin{gathered}
\dot{x}=f_{0}(X, t) X+B u, \quad u=-K(Y) Y \\
=f_{c}(X, t) X \\
f_{c}(X, t)=A_{c} \\
=f_{0}(X, t)-B K(Y) C
\end{gathered}
$$

To select the stable gain, take a Lyapunov function candidate as

$$
V(X)=\frac{1}{2} X^{T} P X, \quad P>0
$$

The derivative of (11) becomes

$$
\begin{aligned}
\dot{V}(X)= & X^{T}\left[f_{0}(X, t)^{T} P+P f_{0}(X, t)\right] X \\
& +u^{T} B^{T} P X+X^{T} P B u
\end{aligned}
$$

If take the control input as

$$
u=-B^{T} P Y
$$

and $Q(X, t)>0$ for all $X \in R^{n}$ and for all $t \geq 0 \quad$ is

$$
f_{0}(X, t)^{T} P+P f_{0}(X, t)=-Q(X, t)
$$

then

$$
\begin{aligned}
\dot{V}(X)= & -X^{T} Q(X, t) X \\
& -X^{T} C^{T} P B B^{T} P X \\
& -X^{T} P B B^{T} P C X \\
= & -X^{T}\left[Q(X, t)+C^{T} P B B^{T} P+P B B^{T} P C\right] X \\
= & -X^{T}\left[f_{c}^{T}(X, t) P+P f_{c}(X, t)\right] X \\
= & -X^{T} Q_{c}(X, t) X, \\
& \quad Q_{c}(X, t)=f_{c}^{T}(X, t) P+P f_{c}(X, t) \\
\leq & -\lambda_{\min }\left\{Q_{c}(X, t)\right\} X^{2} \\
\leq & 0
\end{aligned}
$$

Therefore the stable gain is chosen as

$$
K(Y)=B^{T} P \text { or }=\left[H_{1} C B\right]^{-1} H_{1} f_{0}(X, t)
$$

\section{Control Input}

A corresponding control input is proposed as follows:

$$
u=-K(Y) Y-\triangle K Y-K_{1} S-K_{2} \operatorname{sign}(S)
$$

where $K(Y)$ is a nonlinear output feedback gain satisfying the relationship (10), $\Delta K$ is a switching gain of the state, $K_{1}$ is a feedback gain of the output feedback integral sliding surface, and $K_{2}$ is a switching gain, respectively as

$$
\begin{aligned}
& K(Y)=\left[H_{1} C B\right]^{-1} H_{1} f_{0}(x, t) \\
& \text { or }=B^{T}(x, t) P \\
& \triangle K=\left[\triangle k_{j}\right]
\end{aligned}
$$

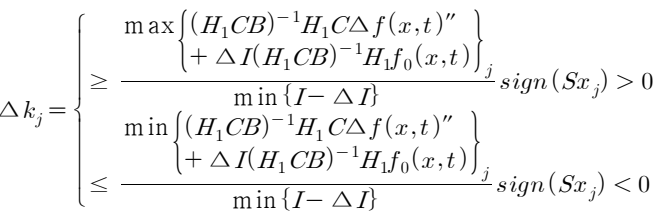

$$
\begin{aligned}
& j=1, \ldots, n \\
& K_{1}>0 \\
& K_{2}=\frac{\max \left\{\left|d^{\prime \prime}(x, t)\right|\right\}}{\min \{I-\Delta I\}}
\end{aligned}
$$

The real sliding dynamics by the proposed control (22) with the output feedback integral sliding surface (8) is obtained as follows: 


$$
\begin{aligned}
\dot{S}= & \left(H_{1} C B\right)^{-1}\left(H_{1} \dot{Y}+H_{0} Y\right) \\
= & \left(H_{1} C B\right)^{-1}\left[\begin{array}{l}
H_{1} C f_{0}(X, t) X \\
+H_{1} C \triangle f(X, t) X \\
+H_{1} C(B+\triangle g(x, t)) u \\
+H_{1} C d^{\prime}(x, t)+H_{0} Y
\end{array}\right] \\
& \quad\left(H_{1} C B\right)^{-1}\left[H_{1} C f_{0}(X, t) X-H_{1} C B K Y+H_{0} Y\right] \\
& +\left(H_{1} C B\right)^{-1}\left[H_{1} C \triangle f(X, t) X\right. \\
& \left.+H_{1} C \triangle g(X, t) K(Y) Y\right] \\
& +\left(H_{1} C B\right)^{-1}\left[H_{1} C(B-\triangle g(X, t))^{*}\right. \\
& \quad\left(-\triangle K Y-K_{1} S-K_{2} \operatorname{sign}(S)\right) \\
& \left.\quad+H_{1} C d^{\prime}(x, t)\right] \\
= & \left.H_{1} C B\right)^{-1}\left[H_{1} C \triangle f(X, t)^{\prime \prime} C X\right. \\
& \left.+H_{1} C \triangle g(X, t) K(Y) Y\right] \\
& -(I-\triangle I) \triangle K(Y) Y \\
& +\left[(I-\triangle I)\left(-K_{1} S-K_{2} \operatorname{sign}(S)\right)\right. \\
& \left.+\quad+d^{\prime \prime}(x, t)\right] \\
= & \left(H_{1} C B\right)^{-1}\left[H_{1} C \triangle f(X, t)^{\prime \prime} Y\right] \\
& +\triangle I\left(H_{1} C B\right)^{-1} H_{1} f_{0}(X, t) Y \\
& -(I-\triangle I) \triangle K(Y) Y \\
& +\left[(I-\triangle I)\left(-K_{1} S-K_{2} \operatorname{sign}(S)\right)\right. \\
& \left.+d^{\prime \prime}(x, t)\right]
\end{aligned}
$$

The closed loop stability by the proposed control input with the output feedback integral sliding surface together with the existence condition of the sliding mode will be investigated in next Theorem 1 .

Theorem 1: If the output feedback integral sliding surface is designed to be stable, i.e. stable design of $K(Y)\left(A_{c}\right)$, the proposed control input with Assumption A1-A8 satisfies the existence condition of the sliding mode on the output feedback integral sliding surface and closed loop exponential stability.

Proof; Take a Lyapunov function candidate as

$$
V(X)=\frac{1}{2} S^{T} S
$$

Differentiating (29) with respect to time leads to and substituting (28) into (30)

$$
\begin{aligned}
\dot{V}(X)= & S^{T} \dot{S} \\
= & S^{T}\left(H_{1} C B\right)^{-1} H_{1} C \triangle f(X, t) \\
& +S^{T} \triangle I\left(H_{1} C B\right)^{-1} H_{1} f_{0}(X, t) Y \\
& -S^{T}[I-\triangle I] \triangle K Y \\
& -S^{T}[I-\triangle I] K_{1} S+S^{T} d^{\prime \prime}(x, t) \\
& -S^{T}[I-\triangle I] K_{2} \operatorname{sign}(S) \\
\leq & -\epsilon K_{1}\|S\|^{2}, \quad \epsilon=\|(I-\triangle I)\| \\
= & -\epsilon K_{1} S^{T} S \\
= & -2 \epsilon K_{1} V(X)
\end{aligned}
$$

From (30), the following equation is obtained as

$$
\begin{aligned}
& \dot{V}(x)+2 \epsilon K_{1} V(x) \leq 0 \\
& V(t) \leq V(0) e^{-2 \epsilon K_{1} t}
\end{aligned}
$$

And the second order derivative of $V(x)$ becomes

$$
\begin{aligned}
\ddot{V}(x) & =\ddot{S} \dot{S}+\ddot{S S} \\
& =(\dot{S})^{2}+S\left(H_{1} C B\right)^{-1}\left(H_{1} \ddot{C X}+H_{0} \dot{C X}\right)<\infty
\end{aligned}
$$

and by Assumption A6 $\ddot{V}(x)$ is bounded, which completes the proof of Theorem 1 .

For practical applications, the discontinuity of the control input (22) must be improved by the modified saturation function

$$
u=-K(Y) Y-K_{1} S-\left[\triangle K Y+K_{2} \operatorname{sign}(S)\right] \frac{S}{|S|+\delta}
$$

\section{Design Example and Simulation Studies}

Consider a third order uncertain more affine nonlinear system with unmatched system matrix uncertainties and matched input matrix uncertainties and disturbance

$$
\begin{aligned}
& {\left[\begin{array}{c}
\dot{x}_{1} \\
\dot{x_{2}} \\
\dot{x_{3}}
\end{array}\right]=\left[\begin{array}{clc}
-3-3 \sin ^{2} x_{1} & 1 & 0 \\
0 & -2 & 1 \\
1+.5 \sin ^{2} x_{2} & 0 & 2+0.4 \sin ^{2} x_{3}
\end{array}\right]\left[\begin{array}{l}
x_{1} \\
x_{2} \\
x_{3}
\end{array}\right]} \\
& +\left[\begin{array}{c}
0 \\
0 \\
2+0.3 \sin (2 t)
\end{array}\right] u+\left[\begin{array}{c}
0 \\
0 \\
\left.d_{1}(X, t)\right)
\end{array}\right] \\
& Y=\left[\begin{array}{lll}
1 & 0 & 0 \\
0 & 0 & 1
\end{array}\right]\left[\begin{array}{lll}
x_{1} & x_{2} & x_{3}
\end{array}\right]^{T} \\
& d_{1}(X, t)=0.7 \sin \left(x_{1}\right)-0.8 \sin \left(x_{2}\right) \\
& +0.2\left(x_{1}^{2}+x_{3}^{2}\right)+1.5 \sin (2 t)+1.5
\end{aligned}
$$

where the nominal matrices $A, B$ and $C$, the unmatched system matrix uncertainties and matched imput matrix uncertainties and matched disturbance are

$$
\begin{aligned}
& f_{0}=\left[\begin{array}{rrr}
-3 & 1 & 0 \\
0 & -2 & 1 \\
1 & 0 & 2
\end{array}\right], B=\left[\begin{array}{l}
0 \\
0 \\
2
\end{array}\right], \quad C=\left[\begin{array}{lll}
1 & 0 & 0 \\
0 & 0 & 1
\end{array}\right] \\
& \Delta f=\left[\begin{array}{ccc}
-3 \cdot \sin ^{2} x_{1} & 0 & 0 \\
0 & 0 & 0 \\
0.5 \sin ^{2} x_{2} & 0 & 0.4 \sin ^{2} x_{3}
\end{array}\right], \\
& \Delta g=\left[\begin{array}{c}
0 \\
0 \\
0.3 \sin (2 t)
\end{array}\right], d(X, t)=\left[\begin{array}{c}
0 \\
0 \\
d_{1}(X, t)
\end{array}\right]
\end{aligned}
$$

The eigenvalues of open loop system matrix A are $-2.6920,-2.3569$, and 2.0489 , hence $\mathrm{A}$ is unstable. The unmatched system matrix uncertainties and matched input matrix uncertainties and matched disturbance satisfy the 


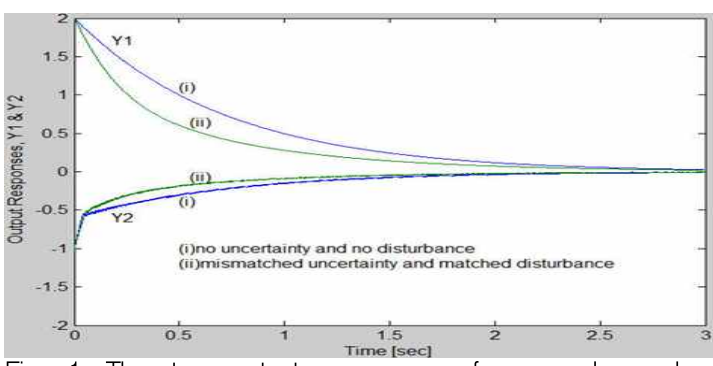

Fig. 1 The two output responses of $y_{1}$ and $y_{2}$ by conventional VSS (i) with no uncertainty and no disturbance and (ii) with mismatched uncertainty and matched disturbance

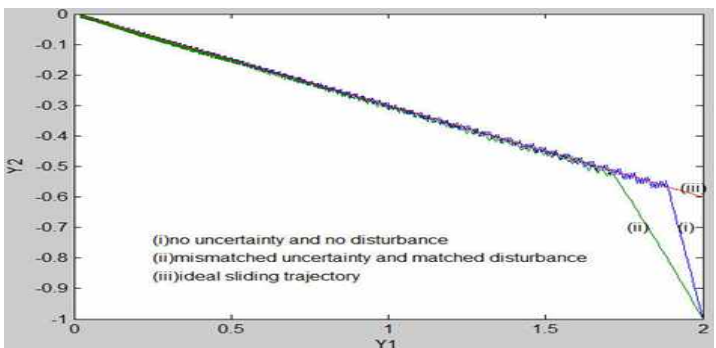

Fig. 2 The three case phase trajectories (i) with no uncertainty and no disturbance, (ii) with mismatched uncertainty and matched disturbance, and (iii)ideal sliding trajectory

assumption A3 as

$$
\begin{aligned}
& \Delta A^{\prime \prime}=\left[\begin{array}{cc}
-3 \cdot \sin ^{2} x_{1} & 0 \\
0 & 0 \\
0.5 \sin ^{2} x_{2} & 0.4 \sin ^{2} x_{3}
\end{array}\right], \\
& \Delta I=0.15 \sin (2 t) \leq 0.15<1 \\
& \text { and } d^{\prime \prime}(X, t)=\frac{1}{2} d_{1}(X, t)
\end{aligned}
$$

To design the output feedback integral sliding surface, $A_{c}$ is designed as

$$
A_{c}=A-B K C=\left[\begin{array}{rrc}
-3 & 1 & 0 \\
0 & -2 & 1 \\
-299 & 0 & -300
\end{array}\right]
$$

in order to assign the three stable pole to $A_{c}$ at -300.0034 and $-2.4983 \pm i 0.8689$. The constant feedback gain is designed as

$$
\begin{aligned}
K C & =2^{-1}\left\{\left[\begin{array}{lll}
1 & 0 & 2
\end{array}\right]-\left[\begin{array}{lll}
-299 & 0 & -300
\end{array}\right]\right\} \\
& =\left[\begin{array}{lll}
300 & 0 & 302
\end{array}\right] \\
& \therefore K=\left[\begin{array}{ll}
150 & 151
\end{array}\right]
\end{aligned}
$$

Then, one find $H_{1}=\left[\begin{array}{ll}h_{11} & h_{12}\end{array}\right]$ and $H_{0}=\left[\begin{array}{ll}h_{01} & h_{02}\end{array}\right]$ which satisfy the relationship (10) as

$$
\therefore h_{11}=0, \quad h_{01}=299 h_{12}, \quad h_{02}=300 h_{12}
$$$$
\text { One select } h_{12}=1 \quad \text { and }
$$

$h_{01}=299$ and $h_{02}=300$. Hence $H_{1} C B=2 h_{12}=2$ is a non zero satisfying $\mathrm{A} 4$. The resultant output

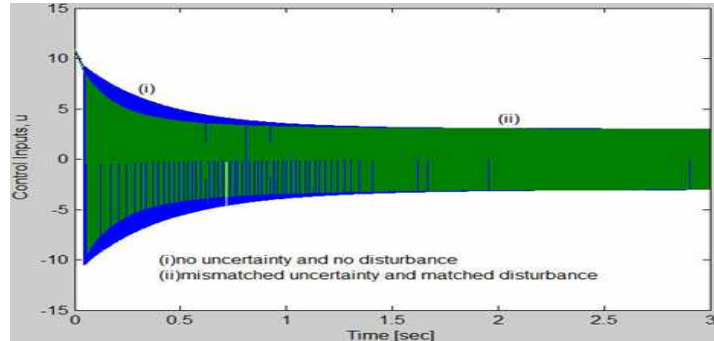

Fig. 3 Two case output feedback integral sliding surfaces (i) no uncertainty and no disturbance and (ii) matched uncertainty and matched disturbance

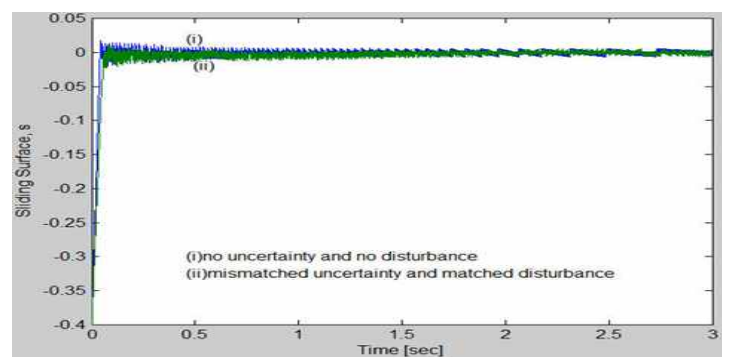

Fig. 4 Two control inputs (i) no uncertainty and no disturbance and (ii) matched uncertainty and matched disturbance

feedback integral sliding surface becomes

$$
\begin{gathered}
y_{01}=\int_{0}^{t} y_{1}(\tau) d \tau \\
S=\frac{1}{2}\left\{\left[\begin{array}{ll}
0 & 1
\end{array}\right]\left[\begin{array}{l}
y_{1} \\
y_{2}
\end{array}\right]+\left[\begin{array}{ll}
299 & 300
\end{array}\right]\left[\begin{array}{l}
y_{01} \\
y_{02}
\end{array}\right]\right\}(=0)
\end{gathered}
$$

where

$$
y_{02}=\int_{0}^{t} y_{2}(\tau) d \tau-y_{2}(0) / 300
$$

The control gains in (22), (24)-(27) are selected as follows:

$$
\begin{aligned}
& \Delta k_{1}=\left\{\begin{array}{r}
1.4 \quad \text { if } S y_{1}>0 \\
-1.4 \quad \text { if } S y_{1}<0
\end{array}\right. \\
& \Delta k_{2}=\left\{\begin{aligned}
1.5 & \text { if } S y_{2}>0 \\
-1.5 & \text { if } S y_{2}<0
\end{aligned}\right. \\
& K_{1}=10.0 \\
& K_{2}=3.0+0.2\left(y_{1}^{2}+y_{2}^{2}\right)
\end{aligned}
$$

The simulation is carried out under $1[\mathrm{msec}]$ sampling time and with $X(0)=\left[\begin{array}{lll}2 & 0.5 & -1\end{array}\right]^{T} \quad$ initial state. For performance comparison, the results of the conventional output feedback VSS are shown in Fig. 1 through Fig. 4. Fig. 1 shows the two output responses of $y_{1}$ and $y_{2}$ (i) with no uncertainty and no disturbance and (ii) with mismatched uncertainty 
Nonlinear Systems with Mismatched Uncertainties and Matched Disturbance

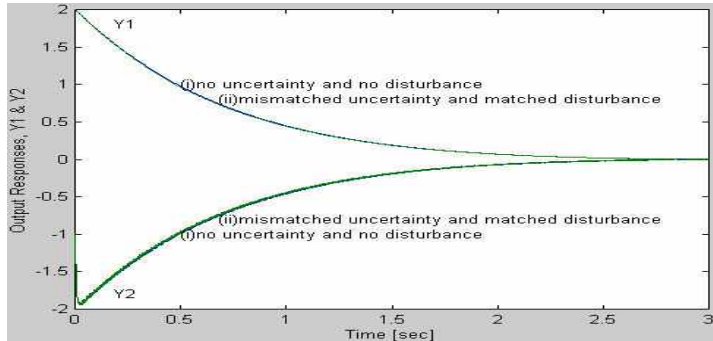

Fig. 5 The two output responses of $y_{1}$ and $y_{2}$ (i) with no uncertainty and no disturbance and (ii) with mismatched uncertainty and matched disturbance

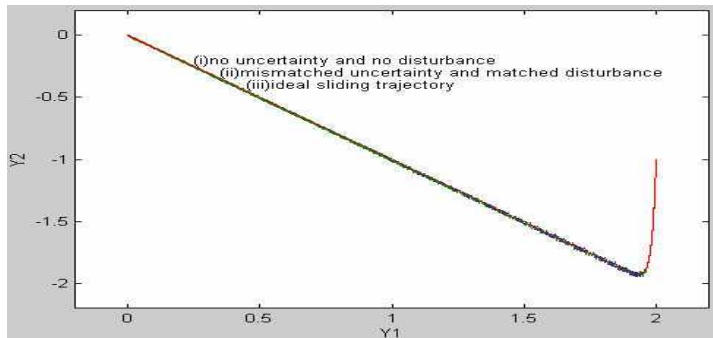

Fig. 6 The three case phase trajectories (i) with no uncertainty and no disturbance, (ii) with mismatched uncertainty and matched disturbance, and (iii)ideal sliding trajectory

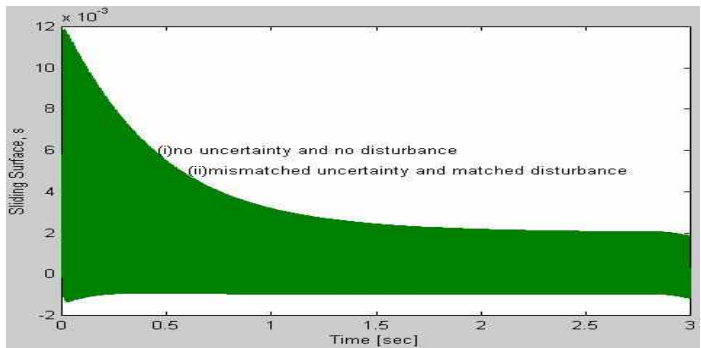

Fig. 7 Two case output feedback integral sliding surfaces (i) no uncertainty and no disturbance and (ii) matched uncertainty and matched disturbance

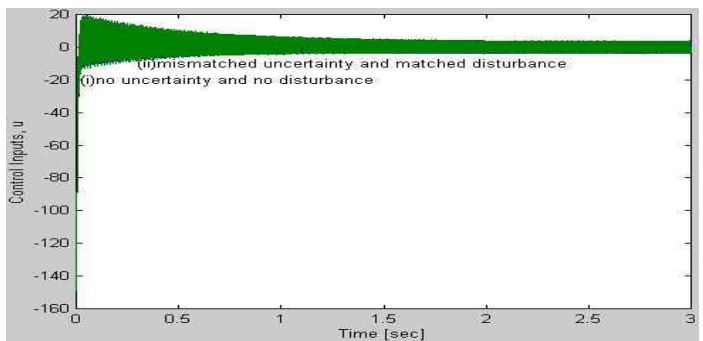

Fig. 8 Two control inputs (i) no uncertainty and no disturbance and (ii) matched uncertainty and matched disturbance

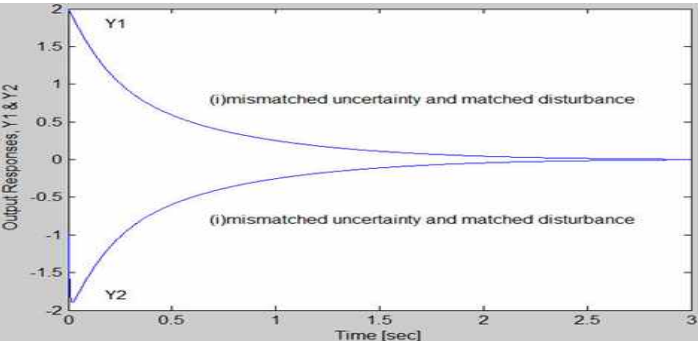

Fig. 9 The two output responses of $y_{1}$ and $y_{2}$ (i) with mismatched uncertainty and matched disturbance

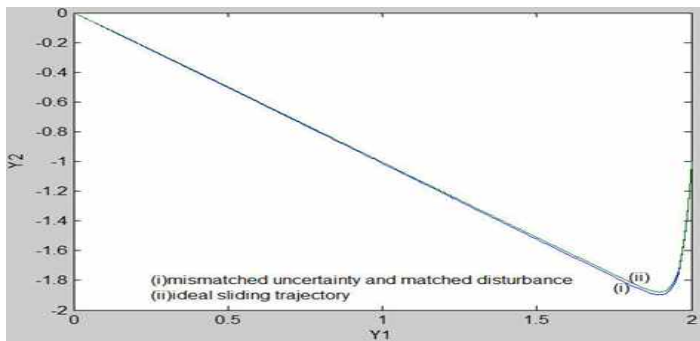

Fig. 10 The two case phase trajectories (i) with mismatched uncertainty and matched disturbance and (ii)ideal sliding trajectory

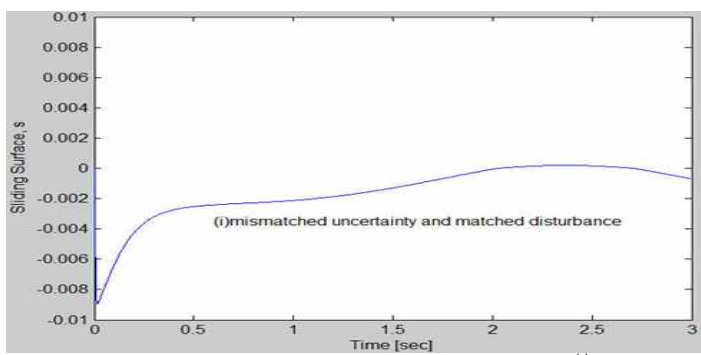

Fig. 11 Output feedback integral sliding surface (i) matched uncertainty and matched disturbance

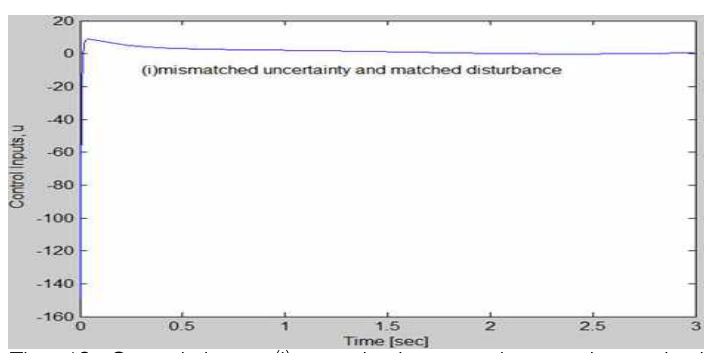

Fig. 12 Control input (i) matched uncertainty and matched disturbance 
and matched disturbance. The each two outputs are different and disturbed by mismatched uncertainty and matched disturbance. The two phase trajectories (i) with no uncertainty and no disturbance and (ii) with mismatched uncertainty and matched disturbance are shown in Fig. 2. There is the reaching phase. During this phase the trajectories are different and disturbed by mismatched uncertainty and matched disturbance. The two case conventional sliding surfaces (i) with no uncertainty and no disturbance and (ii) with mismatched uncertainty and matched disturbance are depicted in Fig. 3. The two control inputs are shown in Fig. 4.

Now the results of the proposed algorithm are shown in Fig. 5 through Fig. 12. Fig. 5 shows the two output responses of $y_{1}$ and $y_{2}$ (i) with no uncertainty and no disturbance and (ii) with mismatched uncertainty and matched disturbance. The each two output is insensitive to the mismatched uncertainty and matched disturbance, hence is almost equal, so that the output can be predicted. The two phase trajectories (i) with no uncertainty and no disturbance and (ii) with mismatched uncertainty and matched disturbance are shown in Fig. 6. There is no reaching phase and each phase trajectory is almost identical also. The two case output feedback integral sliding surfaces (i) with no uncertainty and no disturbance and (ii) with mismatched uncertainty and matched disturbance are depicted in Fig. 7. Fig. 8 shows the two control inputs (i) with no uncertainty and no disturbance and (ii) with mismatched uncertainty and matched disturbance. Because the control inputs in Fig. 8 are discontinuous, for $\delta=0.01$ the continuous approximation is made by (34) with mismatched uncertainty and matched disturbance. Fig. 9 shows the output responses by the continuous input with mismatched uncertainty and matched disturbance. The trajectories are shown in fig. 10. The continuous sliding surface and continuous control input are depicted in Fig. 11. and Fig. 12, respectively for practical application.

From the above simulation studies, the proposed algorithm has superior performance in view of the no reaching phase, predetermined output dynamics, complete robustness, and the prediction of the output, high potential of practical application. From the simulation studies, the effectiveness of the proposed nonlinear SMC is proven.

\section{Conclusions}

In this note, a new robust output feedback nonlinear variable structure controller with an output feedback integral sliding surface is presented based on state dependent nonlinear form for the control of uncertain more affine nonlinear systems with mismatched uncertainties and matched disturbance. After an affine uncertain nonlinear system is represented in the form of state dependent nonlinear system, a systematic design of a new robust nonlinear variable structure controller with the output feedback integral sliding surface is suggested for removing the reaching phase. A corresponding control input is proposed. The closed loop stability by the proposed control input with output feedback integral sliding surface together with the existence condition of the sliding mode on the selected sliding surface will be investigated in Theorem 1 for all mismatched uncertainties and matched disturbance. Through a design example and simulation study, the usefulness of the proposed controller is verified. The output with mismatched uncertainty and matched disturbance is insensitive to the matched uncertainty and disturbance.

\section{References}

[1]M. Vidyasagar, "New Directions of Research in Nonlinear System Theory," Proc. of the IEEE vol.74 no.8 pp.1060-1091, 1986.

[2]P Kokotovic and M. Arcak, "Constructive Nonlinear Control: a Historical Perspective," Automatica, vol.37, pp.637-662, 2001.

[3]B. D. O. Anderson and J. B. More, Optimal Control, Prentice-Hall, 1990.

[4]I. Horowitz, "Survey of Quantitative Feedback Theory(QFT), Int. J. Control, vol.53, no.2 pp.255-291, 1991.

[5]W. J. Rugh and J. Shamma, "Research on Gain Scheduling," Automatica, vol.36, pp.1401-1425, 2000

[6]X. Cai, R. Lin and S. Su, "Robust stabilization for a class of Nonlinear Systems, IEEE CDC pp.4840-4844, 2008.

[7]S. Gutman, "Uncertain dynamical Systems:A Lyapunov Min-Max Approach," IEEE Trans. Autom. Contr, AC-24, no. 1, pp.437-443, 1979.

[8]L. R. Hunt, R. Su, and G. Meyer, "Global Transformations of Nonlinear Systems," IEEE Trans. Autom. Contr, AC-28, no. 1, pp.24-31, 1987.

[9]A Isidori, Nonliear Control System, 2e Springer-Verlag, 
1989

[10J. J. E. Slottine and W Li, Applied Nonlinear Control, Prentice-Hall, 1991.

[11]H. K. Khalil, Nonlinear Systems, 2e Prentice-Hall, 1996.

[12]L. Lijun and X Chengkand, "Robust Backstepping Design of a Nonlinear Output Feedbcak System," IEEE CDC 2008, pp.5095-5099.

[13]K. S. Narendra, "Parameter Adaptive Control-the End...or the Beginning?" Proc. 33rd IEEE CDC, 1994.

[14]Q. Theng and F. Wu, "Lyapunov Redesign of Adpative Controllers for Polynomial Nonlinear systems," Proc. IEEE ACC 2009, pp.5144-5149.

[15]V.I. Utkin,, Sliding Modes and Their Application in Variable Structure Systems. Moscow, 1978.

[16] Decarlo, R.A., Zak, S.H., and Mattews, G.P., "Variable Structure Control of Nonlinear Multivariable Systems: A Tutorial," Proc. IEEE, 1988, 76, pp.212-232.

[17]Young, K.D., Utkin, V.I., Ozguner, U, "A Control Engineer's Guide to Sliding Mode Control," 1996 IEEE Workshop on Variable Structure Systems, pp.1-14

[18] Drazenovic, B.:The invariance conditions in variable structure systems, Automatica, 1969, (5), pp.287-295.

[19]B. C. Toledo and R. C. Linares, "On Robust Regulation via sliding Mode for Nonlinear Systems," System \& Control Letters, vol.24, pp.361-371, 1995.

[20]G. Bartolini and A Ferrara, "On Multi-Input Sliding Mode Control of Uncertain Nonlinear Systems," Proc. IEEE 34th CDC, p.2121-2124, 1995.

[21]X. Y. Lu and S. K. Spurgeon, "Robust Sliding Mode Control of Uncertain Nonlinear System," System \& control Letters, vol.32, pp.75-90, 1997.

[22]G. Bartolini A. Pisano, E. Usai, " Digital Second-Order sliding Mode Control for Uncertain Nonlinear Systems," Automatica, vol.37 pp.1371-1377, 2001.

[23]J. Adamy and A. Flemming, "Soft Variable Structure Control:a survey," Automatica, vol.40, pp.1821-1844, 2004.

[24]Y. Wang, C. Jiang, D. Zhou, F. Gao, "Variable Structure Control for a Class of Nonlinear Systems with Mismatched Uncertainties," Applied Mathematics and Computation, pp.1-14, 2007.

[25]G. Y. Tang, R. Dong, and H. W. Gao, "Optimal sliding Mode Control for Nonlinear System with Time Delay," Nonlinear Analysis: Hybrid Systems, vol.2, pp891-899, 2008.

[26]Y. D. Pan, K. D. Kumar, G. J. Liu, and K. Furuta, "Design of Variable Strucutre Control system with Nonlinear Time Varying sliding Sector," IEEE Trans. Autom. Contr, AC-54, no. 8, pp.1981-1986, 2009.

[27]X. Hu and C. Martin, " Linear Reachability Versus Global Stabilization," IEEE Trans. Autom. Contr, AC-44, no. 6, pp.1303-1305, 1999.

[28]Y. M. Sun, " Linear Controllability Versus Global Controllability," IEEE Trans. Autom. Contr, AC-54, no. 7, pp.1693-1697, 2009.

[29]W. H. Chen, D. J. Ballance, and P. J. Gawthrop, "Optimal Control of Nonlinear System:A Predictive Control Approach," Automatica, vol. 39, pp633-641, 2003

[30]J. H. Lee, "A New Robust Variable Structure Controller for Uncertain Affine Nonlinear Systems with Mismatched Uncertainties, Trans. KIEE vol.59, no.5, pp.945-949, 2010.

[31]J. H. Lee, “A New Robust Integral Variable Structure Controller for Uncertain More Affine Nonlinear Systems with Mismatched Uncertainties," Trans. KIEE vol.59, no.6, pp.1173-1178, 2010.

[32]J. H. Lee, "A New Robust Variable Structure Controller with Nonlinear Integral-Type Sliding Surface for Uncertain Systems with Mismatched Uncertainties and Disturbance," Trans. KIEE vol.59, no.3, pp.623-629, 2010.

[33]J. H. Lee, "A Proof of Utkin's Theorem for the SI Uncertain Integral Linear Case," Trans. KIEE vol.60, no.4, pp.843-747, 2011.

\section{BIOGRAPHY}

Lee Jung-Hoon (Member)

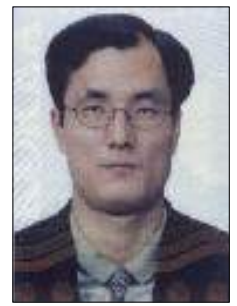
1988 : BS degree in Electronics Engineering, KyeongBook National University. 1990 : MS degree in Control \& Power Electronics Engineering, KAIST. 1995 : PhD degree in Control \& Power Electronics Engineering, KAIST. 1995 present: Professor, Dept. of Control \& Instrum. Eng., Gyeongsang National University. 